\title{
Presurgical evaluation of patients with epilepsy and normal MRI: role of scalp video-EEG telemetry
}

\author{
C A Scott, D R Fish, S J M Smith, S L Free, J M Stevens, P J Thompson, J S Duncan,
} $S$ D Shorvon, W F J Harkness

The National Hospital for Neurology and Neurosurgery, Queen Square, London WC1N 3BG, UK

C A Scott

D R Fish

S J M Smith

$\mathrm{J} M$ Stevens

P J Thompson

J S Duncan

S D Shorvon

W F J Harkness

National Society for Epilepsy, Chalfont St Peter

Buckinghamshire, UK

D R Fish

S J M Smith

$S$ L Free

J M Stevens

P J Thompson

J S Duncan

$S$ D Shorvon

W F J Harkness

Epilepsy Research Group, Institute of Neurology, Queen

Square, London, UK

D R Fish

J S Duncan

S D Shorvon

W F J Harkness

Correspondence to: Miss Catherine Scott, Sir Jules Thorn Telemetry Unit, The National Hospital for Neurology and

Neurosurgery, Queen

Square, London WC1N

3BG, UK. Telephone 0044

171829 8746; fax 0044171

8131230

Received 21 November 1997 and in revised form

19 May 1998

Accepted 29 May 1998

\begin{abstract}
When considering surgery for intractable partial seizures, even with high resolution MRI, some patients do not show structural abnormalities. The aim was to consider whether these patients were likely to proceed to surgical treatment after scalp video-EEG telemetry. All patients undergoing presurgical evaluation at the $\mathrm{Na}$ tional Hospital for Neurology and Neurosurgery between 1995 and 1997 were reviewed and 40 were identified without definite MRI abnormalities. None of 40 disclosed a well localised epileptogenic zone concordant with other tests that would have allowed the patient to proceed directly to surgery. In five of the 40 , evaluation led to a hypothesis that could be tested by intracranial studies; three proceeded to surgery. It is suggested that high quality MRI is performed first when surgical evaluation is undertaken and if negative the patient carefully counselled before proceeding with any investigations, as successful resective surgery is an unlikely outcome in such MRI negative cases.

(7 Neurol Neurosurg Psychiatry 1999;66:69-71)
\end{abstract}

Keywords: magnetic resonance imaging; epilepsy; video-EEG telemetry

Historically epilepsy surgery was carried out using electroclinical features to guide the type and extent of any operative procedure. Recently the advent of high quality MRI usually provides identification of the likely underlying histopathological substrate preoperatively. Nevertheless, patients with medically intractable seizures undergo several expensive and time consuming investigations as part of the assessment for suitability for surgery. ${ }^{1}$ The purpose of this study was to ascertain whether patients in whom high resolution MRI showed no definite lesion were likely to proceed to surgical treatment after scalp video-EEG telemetry.

\section{Patients and methods}

Between January 1995 and January 1997, 222 patients attending the National Hospital for
Neurology and Neurosurgery epilepsy clinics had video-EEG telemetry as part of their investigation for epilepsy surgery.

All patients had high resolution MRI performed using a 1.5 Tesla GE Signa machine, with a protocol for T1 weighted coronal images in thin contiguous slices $1.5 \mathrm{~mm}$ or less and T2 weighted images as described previously. ${ }^{23}$ This allowed volumetric measurements of the hippocampus to be made, $\mathrm{T} 2$ relaxation times to be calculated, and three dimensional reconstruction of the images to look for gyral abnormalities.

Forty of 222 (18\%) patients had normal cortical structures on visual inspection of the MRI by an experienced neuroradiologist (JMS). All of these patients were medically intractable and had a stereotyped seizure pattern and interictal spikes on their EEG. Four of 40 patients were excluded from further analysis because no seizures were recorded despite prolonged monitoring. Volume measurements of the hippocampal structures were made in the remaining 36 patients, and were within the previously established control ranges (mean (2 SD)) in 30 patients. The remaining six showed marginal asymmetries (12-18\%) without definite T2 abnormalities.

Of these 36 patients, 21 were women and 15 were men. The median age of habitual seizure onset was 13 years (range 1 month-37 years) and at the time of study it was 32 years (range $17-48$ years).

Thirty two patients underwent a standardised neuropsychological assessment as part of the presurgical investigation and on the basis of the profile of test scores a judgment was made as to any lateralising and localising significance. The four patients not assessed were not English speakers.

Video - EEG telemetry was performed using a PC based system and software developed in house with up to 64 channels of EEG acquired continuously. Automatic spike and seizure detection programs were utilised and in addition virtually all of the raw EEG data was reviewed. Fifteen patients had repeated studies for the following reasons: (1) because no seizures were recorded initially; (2) to perform ictal Neurolite single positron emission com- 
puted tomography (SPECT) studies (six patients); (3) for invasive EEG monitoring (five patients) with stereotactically implanted intracerebral electrodes, subdural strips, or grids appropriate to the clinical question (Spencer probe electrodes and Wyler subdural strips, Ad-Tech). For descriptive purposes scalp EEGs were divided into five categories based on the ictal EEG changes. ${ }^{45}$

After completion of these investigations, all the patients were reviewed in detail at a multidisciplinary meeting to consider their suitability for surgery or the need for further functional tests or invasive studies.

\section{Results}

The median duration of telemetry recording in these 36 patients was 92 hours (range 17-482 hours). Interictal spikes were temporal in 29 patients (bilateral independent in 23 and unilateral in six), localised extratemporal in four, widespread but confined to one hemisphere in three.

A total of 300 seizures were recorded of which 13 were secondary generalised. Five patients had only one seizure, 16 had between two and five seizures, eight had between six and 10 seizures, and seven $>10$ seizures. In the five patients with only one seizure recorded, the EEG was technically good and the seizure represented their only and habitual seizure type. ${ }^{6}$ From the video recordings 17 of 36 patients were thought likely on clinical grounds to have temporal lobe seizures. ${ }^{7}$

Neuropsychological testing disclosed that whereas the mean level of intellectual ability of the patient group was average, the range was broad (verbal IQ $=93.5$; range 66-117: performance $\mathrm{IQ}=98.1$; range $66-132$ ). Eleven of 36 were considered to have a localised test profile, only two of whom had a lateralised (concordant) ictal EEG. The remainder were judged to show signs of bilateral cerebral disturbance (15 of 36) or were assessed to have no cognitive deficits.

Thirteen of $36(36 \%)$ had non-lateralised/ non-localised ictal EEG onsets. In 11 patients there was insufficient evidence to propose suitable hypotheses about the location and extent of the epileptogenic zone to enable intracranial studies to be carried out. In the remaining two patients, despite temporal type seizure semiology there were other discordant test findings which prevented them from proceeding to intracranial studies.

Three of $36(8.3 \%)$ had bitemporal independent ictal EEG onsets (right and left temporal onsets in different seizures), with temporal seizure semiology. One of these patients proceeded to intracranial studies which showed consistent stereotyped right temporal onsets (maximum in the mesial structures) and underwent resective surgery (histology normal). Two were excluded from further studies, one had evidence of bilateral problems from both the interictal EEG and the neuropsychometric tests, and the remaining patient had psychosocial difficulties, which, together with the clustering of her seizures,
Principal reasons for rejection

Non-localising/lateralising ictal EEG

Discordant interictal and ictal EEG data

Seizure onset localised to eloquent cortex

Psychosocial and psychiatric problems

Bilateral neuropsychological deficits

Patient declined further tests

Multifocal onsets on intracranial EEG

Discordant neuropsychometry and ictal EEG

6
6
5
3

meant that she would be unable to tolerate any more prolonged investigations (table).

Seven of $36(19.4 \%)$ had lateralised scalp EEG changes suggesting a temporal origin (initial changes over the temporal region or subsequent evolving rhythmic temporal theta). ${ }^{8}$ All were offered intracranial studies; two patients have had this done; in one patient temporal and extratemporal multifocal ictal onsets were seen and surgery was considered inappropriate. In the other patient a lateralised neocortical onset was found and surgery was subsequently performed (histology disclosed microdysgenesis only). Of the remaining five patients, one had improved medical control, two were excluded on the grounds of bilateral psychometric deficits, and two declined further investigations after discussion of the risks or benefits.

Seven $(19.4 \%)$ patients had lateralised scalp EEG ictal onset lacking temporal features. One patient proceeded to invasive studies; multifocal temporal and extratemporal ictal onsets were recorded, precluding surgery. Of the remaining six patients, one had improved medical control, three had seizure semiology suggesting extratemporal epilepsy but inadequate localising information to plan an invasive study, one had bilateral deficits on neuropsychometry and scalp EEG, and the other patient declined invasive studies due to the potential risks.

Six patients $(16.7 \%)$ had localised extratemporal ictal EEGs (three frontal, two occipital, and one parietal). One patient proceeded to non-resective surgery after intracranial study. The other five patients had ictal scalp EEG onsets localised to areas of eloquent cortex, or areas thought to be surgically inaccessible and similarly were thought not to be suitable for resective surgery.

In summary, after the multidisciplinary meeting five patients agreed to the recommendation that invasive recordings should be carried out (figure). The remaining 31 patients were rejected from the surgical programme for one or more reasons or they declined further investigations. Overall three of $36 \mathrm{MRI}$ negative patients were eventually offered surgery. One of these underwent a multiple subpial transection of the left sensorimotor cortex. Seizures continued and he died after a seizure 6 months postoperatively. The other two patients have recently had temporal lobe resections after intracranial studies. By comparison, 100 of 182 patients evaluated with MRI abnormalities have already undergone resective surgery despite the complex case mix. 


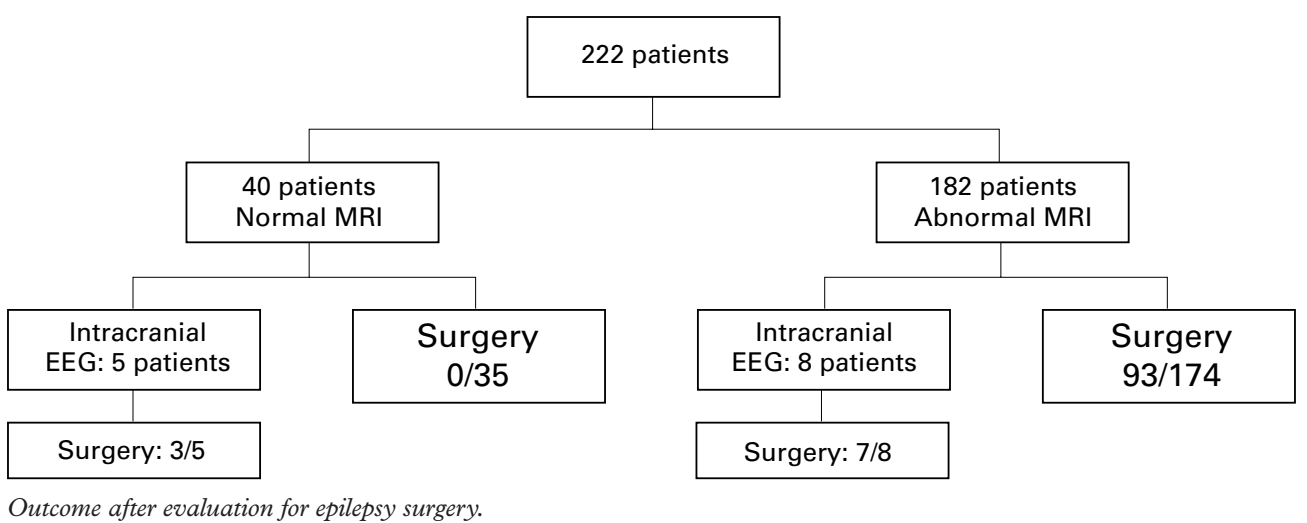

\section{Conclusions}

The finding of lesions (including hippocampal sclerosis) on MRI has greatly increased the number of patients in whom surgical treatment for intractable epilepsy can be successfully carried out. However, despite advances in imaging there remain about $20 \%$ of presurgical patients who have no structural abnormality apparent even utilising tailored MRI protocols. ${ }^{9}$ This study indicates that in such patients intensive and laborious investigation often precludes resective surgery. The MRI negative patients included in this study represent a selected group, taken from a tertiary referral epilepsy practice, who were thought on clinical grounds to be potential surgical candidates. Many other patients attending these clinics would have been MRI negative but were excluded from presurgical evaluation because of other findings, such as multiple seizure types.

All patients were discussed in detail after the scalp telemetry: in only five of 36 patients was it possible to hypothesise a discrete potentially resectable epileptogenic zone that would be amenable to investigation by invasive studies, and many patients had more than one reason for being excluded. Additional functional imaging studies may help to generate such hypotheses in some of the residual patients but to date this has not been our usual experience.

It would seem important when surgery for epilepsy is being considered that high quality MRI using an appropriate dedicated protocol is performed early in the evaluation. If this is negative the patient should be carefully counselled with respect to the reduced chance of eventually proceeding to surgery. Subjecting MRI negative patients to a long and arduous series of investigations may sometimes be unjustified given the risks of antiepileptic drug withdrawal during telemetry and invasive procedures. There is also the detrimental psychological effect of eventual rejection after such efforts and the consequential delay in seeking alternative treatment strategies such as developmental drugs while patients await these inpatient studies.

Although these results relate to practice at a single specialised centre we think that they are generally relevant, given the more widespread introduction of high resolution MRI in other comparable surgical centres.

This work has been funded by the National Hospital for Neurology and Neurosurgery, Queen Square, London WC1N 3BG.

1 Engel J, ed. Surgical treatment of the epilepsies. 2nd ed. New York: Raven Press, 1993

2 Cook MJ, Fish DR, Shorvon SD, et al. Hippocampal volumetric and morphometric studies in frontal and temporal lobe epilepsy. Brain 1992;115:1001-15.

3 Van Paesschen W, Sisodiya S, Connelly A, et al. Quantitative hippocampal MRI and intractable temporal lobe epilepsy. Neurology 1995;45:2233-40.

4 Risinger MW, Engel J, Van Ness PC, et al. Ictal localisation of temporal lobe seizures with scalp sphenoidal recordings. Neurology 1989;39:1288-93.

5 Walczak TS, Radtke RA, Lewis DV. Accuracy and interobserver reliability of scalp ictal EEG. Neurology 1992;42: server re

6 Sum JM, Morrell MJ. Predictive value of the ictal recording in determining localisation of the epileptogenic region by scalp/sphenoidal EEG. Epilepsia 1995;36:1033-40.

7 Engel J. Update on surgical treatment of the epilepsies. Neurology 1993;43:1612-17.

8 Ebersole JS, Pacia SV. Localisation of temporal lobe foci by ictal EEG patterns. Epilepsia 1996;37:386-99.

9 Duncan JS. Imaging and epilepsy. Brain 1997;120:339-77. 